\title{
Wirtschaftlichkeit von Innovativen Wandkonstruktionen im Wohnungsbau
}

\author{
Emanuel Stocker $\cdot$ Christopher Fahr $\cdot$ David Koch
}

Eingegangen: 27. Februar 2017 / Angenommen: 23. November 2018 / Online publiziert: 5. Dezember 2018

(C) Der/die Autor(en) 2018

Zusammenfassung Der Einsatz innovativer Materialien, die eine oder mehrere Eigenschaften verbessern, steht oder fällt in der praktischen Anwendung mit der Wirtschaftlichkeit. Diese Arbeit analysiert den Einsatz innovativer Wandkonstruktionen im Wohnungsbau. Im Vergleich zu konventionellen Konstruktionen verursachen innovative Wandkonstruktionen (z.B. Vakuumdämmsystem) höhere Aufwendungen, besitzen aber im Gegenzug eine deutlich geringere Konstruktionsbreite und erzeugen so einen Flächengewinn.

Anhand eines Referenzgebäudes, das auf realen Gebäuden basiert, erfolgt eine Gegenüberstellung hinsichtlich Außenwänden und Innenwänden. Es zeigt sich, dass gegenüber dem Mehraufwand von $\sim 128.000 €$ auf der anderen Seite ein Nutzungsflächengewinn (NUF 1) von $65,76 \mathrm{~m}^{2}$ (7,7\% Steigerung) gegenübersteht. D. h. bei dem bestehenden Referenzgebäude mit zwölf Einheiten könnte eine zusätzliche Wohneinheit bei gleichbleibender Brutto-Grundfläche geschaffen werden. Die Wirtschaftlichkeit dieser Maßnahmen orientiert sich an den Immobilienpreisen und sofern dieser über der Kostenschwelle von $1956 € / \mathrm{m}^{2}$ Nutzungsfläche liegt, können diese Maßnahmen als wirtschaftlich von Vorteil angesehen werden. Aufgrund der Unsicherheit in der Annahme einiger Berechnungsdaten wird abschließend eine Simulation durchgeführt. Die Ergebnisse daraus zeigen eine höhere Bandbreite, je-

\footnotetext{
E. Stocker $(\bowtie) \cdot$ D. Koch

Institut für Facility Management \& Immobilienwirtschaft, Fachhochschule Kufstein Tirol, Andreas Hofer Straße 7, 6330 Kufstein, Österreich

E-Mail: Emanuel.Stocker@fh-kufstein.ac.at

D. Koch

E-Mail: David.Koch@fh-kufstein.ac.at

C. Fahr

ÖBB Immobilienmanagement GmbH, Hochbau \& Liegenschaftsentwicklung Salzburg \& Oberösterreich, Südtirolerplatz 1, 5020 Salzburg, Österreich

E-Mail: Christopher.Fahr@oebb.at
} 
doch aufgrund der Annahme von besseren Flächenverhältnissen eine wirtschaftlich positivere Situation.

Schlüsselwörter Innovative Materialien · Wandkonstruktionen · Wirtschaftlichkeit · Wohnungsbau

\section{Profitability of innovative wall constructions for residential buildings}

Abstract Innovative materials improve one or more specifications of a building and for the practical application the profitability is one of most important criteria. This paper analyses alternative and innovative wall construction, especially for multifamily buildings. Based on a reference building, the geometric building characteristics as well as the structural features of the wall structures serve as the subject of the initial situation of the analyses. As alternative wall constructions, superstructures were chosen, which can basically reduce the construction area. Such wall constructions, for example in the form of vacuum panels, generate higher expenses and parallel more living space compared to the conventional constructions. Therefore, it is necessary to investigate if such an alternative is economically. The results show that the living space in total can be increased by approximately $7.7 \%$ on the basis of the alternatives. From the economic perspective the profitability depends on the real estate prices and if the price is higher than the cut-off point of about $1,956 € / \mathrm{m}^{2}$ living space the alternative is economically advantageous. Especially from the alternative of the outer wall construction, in the form of prefabricated concrete walls and vacuum insulation panels, the greatest potential is achieved. Due to the uncertainty in the assumption of some data, a simulation is finally carried out. The results show a higher bandwidth of the economic result, but within a better surface ratio a more favourable economic situation.

Keywords Innovative Materials · Wall Constructions · Profitability · Residential Buildings

\section{Einleitung}

Die Wirtschaftlichkeit eines Bauvorhabens wird zunehmend durch dessen Planung im Hinblick auf die Geometrie und auch die Auswahl der Konstruktionen bestimmt. Die Ausnutzung der maximal zu errichtenden Grundfläche, welche vom öffentlichen Baurecht vorgegeben wird, ist dabei ebenso relevant, wie die Ausnutzung der maximal verfügbaren Nutzungsfläche (NUF 1: Wohnen und Aufenthalt) (DIN 277 2016). Dies bedeutet, dass die äußere Grenze der bebaubaren Grundfläche im Regelfall feststeht und die innere Nutzungsfläche sich aus der Planung und der Wahl der Konstruktionen ergibt. Jede dieser Konstruktionen hat entsprechende Anforderungen zu erfüllen, wie bspw. statische Tragfähigkeit, Wärme-, Feuchte-, Schallschutz, oder lediglich die räumliche Trennung. Betrachtet man konventionelle Konstruktionen aus Außenwänden, dann sind Aufbaudicken von ca. $50 \mathrm{~cm}$ üblich. An dieser Stelle gilt es zu untersuchen, inwieweit Konstruktionen, die schlanker im Aufbau 
sind und somit eine höhere Nutzungsfläche generieren, generell aber höhere Kosten verursachen, auch wirtschaftlicher sind. D.h. inwieweit kann der Flächengewinn an Nutzungsfläche (bspw. höherer Mietertrag) die höheren Kosten der baulichen Konstruktion rechtfertigen. Auf die Effizienz der Flächennutzung machte bereits Kalusche (2011) bezugnehmend auf die Kennwerte aus dem Baukosteninformationszentrum (BKI Baukosteninformationszentrum 2010) aufmerksam. Speziell die Kennwerte der Konstruktions-Grundfläche (KGF) im Verhältnis zur Brutto-Grundfläche (BGF) bzw. deren hohen Bandbreite sind dort benannt worden. Der Anteil der KGF an der BGF liegt bei Mehrfamilienhäusern ab sechs Wohneinheiten durchschnittlich zwischen 14,5 und 15\% (Kalusche 2011). Die Kennwerte aus der aktuelleren Sammlung aus dem Jahr 2017 (BKI Baukosteninformationszentrum 2017) für die Objektkategorie „Mehrfamilienhäuser 6-19 Einheiten“ ergeben einen Anteil von 14,6 bis 18,0\% (KGF/BGF), im Mittelwert 16,2\%. Mit dem Bezugswert der Nutzungsfläche (NUF) können Kennwerte KGF/NUF von 20,7 bis 27,1\% (im Mittel 23,4\%) entnommen werden. Aus den Kennwerten ergibt sich bereits ein hohes Potential auf Grundlage der Wahl und Anordnung der KGF. Dieser Anteil kann über die Verwendung von modernen Konstruktionsmaterialien zugunsten der veräußerbaren Nutzungsfläche signifikant vermindert werden. Aus diesem Ansatz geht das Ziel dieser Untersuchung hervor, indem an einem realitätsnahen Referenzgebäude untersucht wird, ob und wann innovativere Konstruktionsaufbauten (hier: Vakuumpaneele - Vacuum insulation panels - kurz: VIPs), die oftmals mit höheren Investitionskosten verbunden sind, durch den Gewinn an Nutzungsfläche wirtschaftlich vorteilhaft sind.

\section{Aktuelle Forschung}

In der Forschung steht die Wirtschaftlichkeit hinsichtlich alternativer bzw. innovativer Bauelemente (zur Einsparung von KGF) nur bedingt im Fokus. Jelle (2011) befasste sich mit unterschiedlichen traditionellen und innovativen Dämmstoffen hinsichtlich der Unterschiede in der Wärmeleitfähigkeit. In der Untersuchung wurde auch das Potential der Flächengewinne durch den Einsatz von Vakuumpaneelen analysiert. Die Analyse basierte auf dem Vergleich herkömmlichen Dämmmaterials mit einer Stärke von $35 \mathrm{~cm}$ und einer VIP Dämmung mit $6 \mathrm{~cm}$ Stärke. Aus der Gegenüberstellung geht hervor, dass die VIP Dämmung ab bei einem Wert von ca. $2200 € / \mathrm{m}^{2}$ Nutzungsfläche profitabel ist. Die Ergebnisse basieren auf einem Beispielgebäude mit den Abmessungen von $10 \mathrm{~m} \times 10 \mathrm{~m} \times 2,5 \mathrm{~m}$. Naji et al. (2014) untersuchten drei unterschiedlichen Leichtbauweisen in der Türkei. Die Studie befasste sich mit den Konstruktionskosten und der Lebensdauer. Flächengewinne etc. wurden hier jedoch nicht berücksichtigt. Der Einfluss der Fläche bzw. des Flächen-Volumenverhältnisses ist vor allem im Fokus von Studien, die sich mit der Energieeffizienz auseinandersetzen. Exemplarisch kann hierbei die Untersuchung von McKeen und Fung (2014) genannt werden. Hierbei wurde der Zusammenhang zwischen der Geometrie, Lage und Gebäudekriterien im Hinblick auf den Energieverbrauch analysiert. Sev und Zgen (2009) untersuchten die Flächeneffizienz von Bürogebäuden als Hochhäuser. Sie zeigen auf, dass die Flächeneffizienz und die 
daraus resultierende Optimierung der Mieteinnahme sich meist nicht mit anderen Effizienzzielen (bspw. Konstruktion, Energie; Betriebskosten) vereinbaren lässt.

Eine Studie an der TU Wien (Kolbitsch et al. 2008) im Auftrag der Wirtschaftskammer Österreich befasste sich mit Wirtschaftlichkeitsparametern im geförderten Wohnungsbau in Wien. Ziel der Studie war die Erhebung und Bekanntmachung von Referenzwerttabellen, die u.a. als Grundlage für einen Planungswettbewerb dienen können. Als Referenzwerte wurden Kennwerte zur Einordnung der Flächen-, Raum und Fassadeneffizienz gebildet. Bei der Konstruktions-Grundfläche kann der Kennwert BGF/NGF (Netto-Grundfläche) herangezogen werden und hierzu wurde ein Referenzbereich von 1,10 bis 1,20 definiert. Dies bedeutet, dass Projekte in diesem Rahmen als konstruktiv wirtschaftlich zu deuten sind.

Betrachtet man die Publikationen zu ,innovativen Konstruktionen“, so zeigt sich, dass diese den Fokus auf bauphysikalische Eigenschaften sowie energieeffizientes und ökologisches Bauen legen. Eine Darstellung hinsichtlich der Flächeneinsparung fehlt. Bspw. bei Vakuumpaneelen setzte sich bereits 2004 Wakili et al. mit der Wärmeleitfähigkeit von Vakuumpaneelen auseinander (Wakili et al. 2004). Schwab et al. verfassten ebenfalls bereits 2005 eine Arbeit zu Wärmebrücken in Vakuumdämmungen (Schwab et al. 2005). 2010 setzten sich Tenpierik und Cauberg in ihrer Arbeit „Encapsulated vacuum insulation panels: theoretical thermal optimization“ mit der idealen Dicke und Verwendung von VIPs auseinander (Tenpierik und Cauberg 2010). Johansson stellt in seinem Bericht aus 2012 einen Überblick zu bislang untersuchten Bereichen mit der Verwendung von Vakuumdämmungen dar (Johansson 2012). Yamankaradeniz schrieb 2015 eine Arbeit über das Thema betreffend Kondensation in Wänden welche mit VIPs gedämmt wurden (Yamankaradeniz 2015). Gubbels et al. schrieben ebenfalls 2015 eine Arbeit zur Haltbarkeit von Vakuumdämmungen (Gubbels et al. 2015).

Der Forschungsstand zeigt, dass Untersuchungen hinsichtlich innovativer Bauelemente, Flächeneinsparung und die daraus resultierende Wirtschaftlichkeit nur bedingt umfassend dargestellt werden.

\section{Ziel und Methodik der Untersuchung}

Ziel der Untersuchung ist es, festzustellen, ab welchem Betrag (Verkaufspreis) pro $\mathrm{m}^{2}$ Nutzungsfläche (NUF 1) eine alternative und auch innovative Wandkonstruktion, die eine Flächengewinnung erzeugt, wirtschaftlich von Vorteil ist. Konkret soll bei konventioneller Bauweise (Massivbauweise) eine Alternative für jede Schicht des Aufbaus von Innen- und Außenwänden gefunden und wirtschaftlich analysiert werden. Als Herangehensweise zur Untersuchung der Ziele auf Grundlage der angewandten Forschung wird eine Fallstudie durchgeführt. Für die Fallstudie wird ein Referenzgebäude auf Basis realer Gebäudedaten definiert. Dabei werden die Planunterlagen von den 14 realen Projekten gegenübergestellt und ein durchschnittliches Referenzgebäude abgeleitet. Die konventionellen Konstruktionen stammen dabei ebenfalls von Referenzgebäuden, die innovativeren Konstruktionen anhand Erhebungen aus der Literatur bzw. produzierenden Herstellern. Die Kostendaten stammen einerseits aus Kennwertesammlungen, wie BKI (BKI Baukosteninforma- 
tionszentrum 2017) und andererseits von Angebotspreisen der Hersteller. Sämtliche in der Arbeit angegebenen Kostenwerte sind in netto, ohne Umsatzsteuer. Um die Veränderung der Nutzungsfläche zu erfassen, wurde das Referenzgebäude, inkl. der Varianten mit den unterschiedlichen Konstruktionen in CAD erstellt. Auf Grundlage dieser Berechnungen lässt sich jede einzelne Maßnahme (Variante) in Form einer Kosten-Nutzen-Analyse untersuchen und als Ergebnis wird ein sog. BreakEven Point ermittelt. Der Break-Even stellt damit den Schwellenwert zu den Immobilienpreisen dar. Dies bedeutet, liegt der Preis über dem Schwellenwert, ist die Variante wirtschaftlich von Vorteil.

\section{Referenzgebäude}

Das Referenzgebäude wurde von 14 realen Projekten, gemeinnütziger Bauträger abgeleitet. Dazu wurden jeweils die BGF, die NUF, die Anzahl der Wohneinheiten und die Außenwandfläche $(\mathrm{AwFl})$ erhoben. Die deskriptiv ermittelten Daten und Kennwerte sind in Tab. 1 angeführt.

Betrachtet man die gebildeten Kennwerte, so ist ersichtlich, dass die Streuung gemessen an der Standardabweichung der NUF/BGF im Vergleich zur NUF/AwFl erheblich geringer ist. Dies kann darauf zurückgeführt werden, dass die Flächeneffizienz im Grundriss höher ist, als die Effizienz der Gebäudekörper, bzw. der Kompaktheit. Die Wahl des Referenzgebäudes fiel auf das Projekt, welches den Mittelwerten am nächsten lag. Das daraus definierte Referenzgebäude ist 3-geschossig, mit 13 Wohneinheiten und besitzt eine BGF von $1173 \mathrm{~m}^{2}$. Die grafische Visualisierung ist in Abb. 1 ersichtlich. Balkone und Terrassen sind in der Darstellung nicht enthalten, da diese von der Untersuchung ausgenommen sind.

Abb. 2 stellt den Grundriss (ohne Maßstab) des Erdgeschosses dar. Wie dieser zeigt, weist das Gebäude eine L-Form mit verschiedenen Aussparungen aus. Die hellgraue Fläche stellt die für den Verkauf relevante Nutzungsfläche dar. Der Erschließungskern in der Mitte zählt zu den Verkehrsflächen und wird demnach nicht berücksichtigt. Im Erdgeschoss befinden sich fünf Wohneinheiten mit ca. $332 \mathrm{~m}^{2}$ Nutzungsfläche.

Tab. 1 Deskriptive Statistik der erhobenen Gebäudedaten (Eigene Darstellung)

\begin{tabular}{lllll}
\hline Gebäudedaten (gerundet) & Min & MW & Max & STABW \\
\hline Anzahl der Wohneinheiten & 5 & 12 & 15 & 3 \\
Brutto-Grundfläche (BGF) $\left[\mathrm{m}^{2}\right]$ & 545 & 1160 & 1450 & 260 \\
Nutzungsfläche (NUF) $\left[\mathrm{m}^{2}\right]$ & 385 & 847 & 1057 & 194 \\
NUF/BGF & 0,69 & 0,73 & 0,76 & 0,02 \\
Außenwandfläche (AwFl) $\left[\mathrm{m}^{2}\right]$ & 526 & 936 & 1273 & 194 \\
NUF/AwFl & 0,67 & 0,91 & 1,16 & 0,14 \\
\hline
\end{tabular}




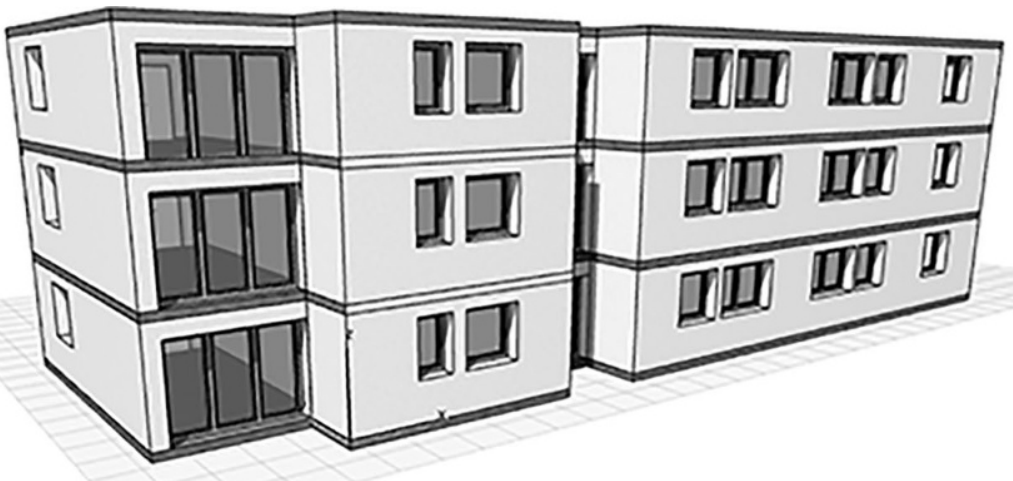

Abb. 1 Visualisierung Referenzgebäude. (Eigene Darstellung)

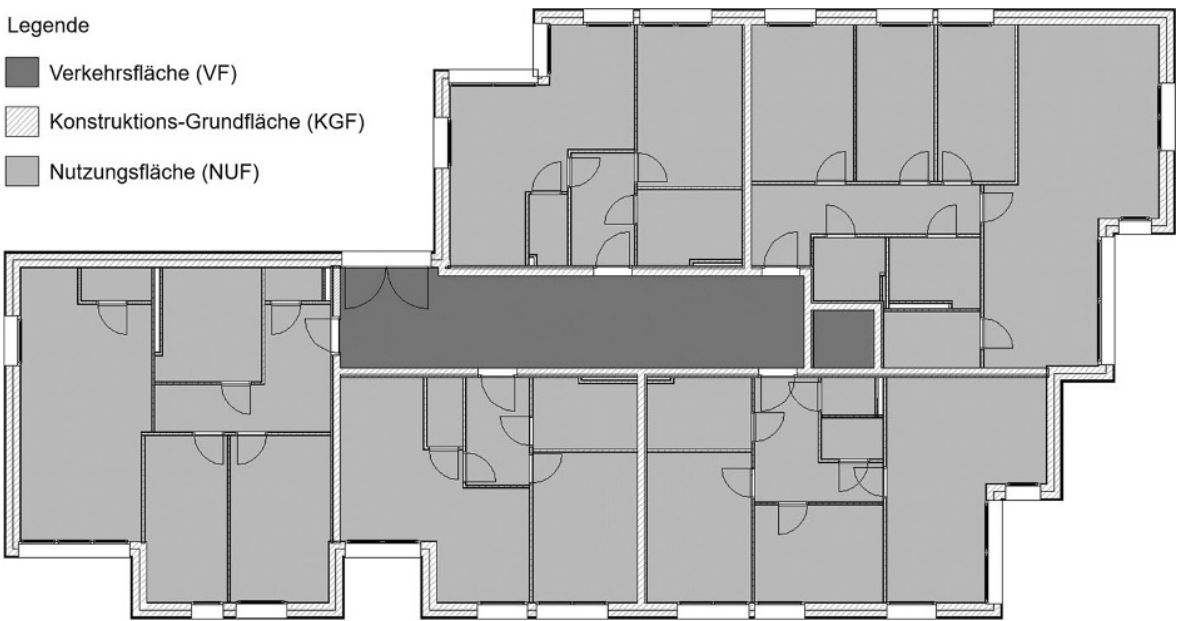

Abb. 2 Grundriss Erdgeschoss. (Eigene Darstellung)

\subsection{Gebäudedaten - Referenzgebäude}

Die geometrischen Gebäudedaten zum Referenzgebäude sind in Tab. 2 zusammengefasst. Den Gebäudedaten kann entnommen werden, dass das Referenzgebäude einen Anteil der Nutzungsfläche (NUF) zur BGF von 0,72 aufweist. Der Anteil der KGF zur BGF beträgt hierbei $17,6 \%$. Vergleichend zu den eingangs erwähnten Kennwerten mit einem Durchschnitt von 16,4\% kann an dieser Stelle angemerkt werden, dass die Wanddicken u. a. auf die energetischen Anforderungen zurück zu führen sind. Dies kommt daher, dass die erhobenen Referenzen im Alpenraum liegen und demnach energetisch höhere Anforderungen haben. 
Tab. 2 Daten und Kennzahlen Referenzgebäude. (Eigene Darstellung)

\begin{tabular}{ll}
\hline Gebäudedaten - Generell & 13 \\
Anzahl der Wohneinheiten & 3 \\
Geschossanzahl & $444,63 \mathrm{~m}^{2}$ \\
Bebaute Fläche & \\
Grundflächen und Kennwerte & $1173,21 \mathrm{~m}^{2}$ \\
Brutto-Grundfläche (BGF) & $849,62 \mathrm{~m}^{2}$ \\
Nutzungsfläche (NUF) & $206,85 \mathrm{~m}^{2}$ \\
Konstruktions-Grundfläche (KGF) & 0,724 \\
NUF/BGF & 0,176 \\
KGF/BGF & \\
Wandflächen & $918,14 \mathrm{~m}^{2}$ \\
Außenwandfläche (AwFl) & $551,61 \mathrm{~m}^{2}$ \\
Außenwandfläche konstruktiv (AwFl_ko) & $\mathbf{a}$ \\
Innenwandfläche tragend (IwFl_tr) & $354,04 \mathrm{~m}^{2}$ \\
Innenwandfläche nicht tragend (IwFl_ntr) & $830,25 \mathrm{~m}^{2}$ \\
\hline
\end{tabular}

a'Ohne Öffnungen (Fenster und Türen)

\subsection{Wandaufbauten - Referenzgebäude}

Neben den generellen Strukturmerkmalen wurden auch die Konstruktionsmerkmale für Außenwände-tragend, Innenwände-tragend und Innenwände-nicht tragend erhoben. Sämtliche der ausgewerteten Gebäude wurden mit Stahlbeton (Ortbeton oder Mantelbeton) errichtet. Lediglich ein Gebäude hatte eine Dicke der Betonwand von $18 \mathrm{~cm}$, die restlichen Gebäude hatten durchgehend $20 \mathrm{~cm}$ dicke Stahlbetonwände. Auf Basis dieser Daten wurde für das Referenzgebäude eine Tragschicht aus Stahlbeton mit $20 \mathrm{~cm}$ festgelegt. Auch bei der Dämmung waren die Materialien und Dicken sehr ähnlich. Alle Gebäude wurden mit einem Vollwärmeschutzsystem (mit Expandiertem Polystyrol - EPS) in Dicken von 14 bis $22 \mathrm{~cm}$ EPS versehen, durchschnittlich waren es $20 \mathrm{~cm}$. Die Dämmschicht des Referenzgebäudes wurde daher mit $20 \mathrm{~cm}$ EPS festgelegt. Zusammenfassend besteht beim Referenzgebäude die $\mathrm{Au}-$ ßenwand (AW) aus einer Stahlbetonkonstruktion mit außenliegendem Wärmedämmverbundsystem, welche innenseitig verputzt ist. Die tragenden Innenwände (IW_tr)

Tab. 3 Wandaufbau und Aufbaustärken [in cm] Referenzgebäude. (Eigene Darstellung)

\begin{tabular}{|c|c|c|c|c|c|c|}
\hline Bauteil & AW & & IW_tr & & IW_ntr & \\
\hline - & Innenputz & $1,5 \mathrm{~cm}$ & Innenputz & $1,5 \mathrm{~cm}$ & $\begin{array}{l}\text { Gipskartonplatten } \\
\text { (2-fach) }\end{array}$ & $2,5 \mathrm{~cm}$ \\
\hline- & Stahlbeton & $20 \mathrm{~cm}$ & Stahlbeton & $20 \mathrm{~cm}$ & $\begin{array}{l}\text { Installationsebene } \\
\text { (Dämmung) }\end{array}$ & $5,0 \mathrm{~cm}$ \\
\hline - & $\begin{array}{l}\text { Dämmung } \\
\text { (EPS) }\end{array}$ & $20 \mathrm{~cm}$ & $\begin{array}{l}\text { Vorsatzschale } \\
\text { Gipskartonplat- } \\
\text { ten }\end{array}$ & $7,5 \mathrm{~cm}$ & $\begin{array}{l}\text { Gipskartonplatten } \\
\text { (2-fach) }\end{array}$ & $2,5 \mathrm{~cm}$ \\
\hline- & Außenputz & $0,5 \mathrm{~cm}$ & - & - & - & - \\
\hline Summe: & $42 \mathrm{~cm}$ & & $29 \mathrm{~cm}$ & & $10 \mathrm{~cm}$ & \\
\hline
\end{tabular}


bestehen ebenfalls aus Stahlbeton, auf einer Seite verputzt und auf der anderen Seite mit einer Gipskartonvorsatzschale versehen. Die nicht tragenden Innenwände (IW_ntr) bestehen durchgehend aus einer doppelt beplankten Trockenbauwand. Die einzelnen Aufbauten und deren Schichtstärken sind in Tab. 3 zusammengefasst.

Das Referenzgebäude hat somit eine Außenwand mit $42 \mathrm{~cm}$, tragende Innenwände mit $29 \mathrm{~cm}$ und nicht tragende Innenwände mit $10 \mathrm{~cm}$ Aufbaustärke. Diese Aufstellung bildet neben dem eigentlichen Gebäude die Definition des Referenzgebäudes anhand der Quantität und Qualität im Bereich der Wandaufbauten. Diese Festlegung dient im Weiteren der Ermittlung der Bauwerkskosten.

\section{Alternative - Innovative Wandsysteme}

Unter ,alternativ“ werden in diesem Zusammenhang Maßnahmen behandelt, die die Wanddicke reduzieren, ohne die relevante Funktion zu beeinträchtigen. Als relevante Funktion kann dabei die statische Tragfähigkeit, oder die Wärmeleitfähigkeit verstanden werden. Ebenso sind in dieser Hinsicht die akustischen und brandschutztechnischen Merkmale nur kaum verändert und im Hinblick der bautechnischen Erfordernisse überprüft worden. Als innovative Bauelemente werden nachfolgend im Wesentlichen zwei Elemente, zum einen Vakuumpaneele (Vacuum insulation panels - VIPs) und zum anderen Betonfertigteile mit höherwertiger Betongüte angesehen.

\subsection{Außenwände}

Als alternative Materialien zu dem erhobenen konventionellen und statisch-konstruktiven Material Beton können Betonfertigteile mit höherwertiger Betongüte verwendet werden, um so die Dimensionen der Konstruktionen zu reduzieren. Die höhere Betongüte bringt demnach auch höhere Kosten mit sich, die nachfolgend mitberücksichtigt werden. Als Alternative zu den konventionellen Dämmsystemen können VIP in Betracht gezogen werden. Dieser Dämmstoff verfügt über eine Wärmeleitfähigkeit von 0,0047 W/mK (Laborwert). Zum Vergleich liegt dieser beim konventionellen Dämmstoff EPS zwischen 0,035 und 0,40 W/mK (Pfau 2013). Dies bedeutet, dass mit einer $4 \mathrm{~cm}$ VIP ein U-Wert von $0,175 \mathrm{~W} / \mathrm{m}^{2} \mathrm{~K}$ erreicht werden $\mathrm{kann}$, was dem Wert einer etwa $20 \mathrm{~cm}$ dicken EPS-Dämmung entspricht. Von der Konstruktion werden die VIP vielfach als hinterlüftete Fassade ausgeführt, jedoch kann der Aufbau auch mit klassischer Putzfassade ausgeführt werden. Die Dämmplatten verfügen in diesem Fall über eine Putzträgerplatte aus Zementfasern (Fermacell o.glw.) in der Dicke von $15 \mathrm{~mm}$. Innenseitig ist als Alternative zum Referenzbauteil die Verwendung einer Verspachtelung vorgesehen, um so die Bauteildicke zu reduzieren. Bei sehr ebenen Untergründen, wie beispielsweise Rigips-Wänden oder Beton-Fertigteilen, ist nicht zwingend ein Innenputz notwendig. An dieser Stelle kann es ausreichend sein, die wenig vorhandenen Unebenheiten zu verspachteln (Pfau 2013). Dies ist wesentlich weniger aufwändig und mit geringeren Kosten verbunden. Die genaue Vorgangsweise und die nötigen Schritte hängen dabei vom jeweiligen Untergrund ab (Rupp 2009). Auf der anderen Seite werden jedoch $1,5 \mathrm{~cm}$ Innenputz bei dieser Alternative eingespart (Krass et al. 2009). Die ermittelten Kosten für die alternative 
Außenwand liegen bei $390,50 € / \mathrm{m}^{2}$ Wandfläche. Der konventionelle Wandaufbau beim Referenzgebäude liegt unter der Hälfte bei $181,24 € / \mathrm{m}^{2}$ Wandfläche.

\subsection{Innenwände - tragend und nicht tragend}

Bei den tragenden Innenwänden kann ebenfalls auf die Alternative der Außenwand in Form von Fertigteilelementen mit höherer Betongüte sowie dem Verspachteln zurückgegriffen werden. Die Vorsatzschale aus Trockenbaukonstruktion des Referenzaufbaus wird in diesem Fall beibelassen, damit die akustischen Anforderungen erfüllt werden. Da bei den nicht tragenden Innenwänden die Schallschutzanforderungen nicht so hoch, wie bei Wohnungstrennwänden (in diesem Fall auch die tragenden Innenwände) sind, ergeben sich dennoch Alternativen zur Referenzwand. Zum einem kann die Innenwand als Ziegelmauerwerk mit einer Stärke von $5 \mathrm{~cm}$ und beidseitig verputzt, d.h. in Summe $8 \mathrm{~cm}$ Wandstärke ausgeführt werden. Zum anderen ist die Ausführung einer Trockenbauwand als Ständerkonstruktion mit jeweils einfacher Beplankung möglich. In diesem Fall ergibt sich eine Stärke von gesamt 7,5 cm. Da die Alternative mittels Ziegelmauerwerk im Vergleich zur einfach beplankten Trockenbauwand in Aufwand und Platzbedarf höher ausfällt, wird nachfolgend die Variante mit der einfachen Gipskartonständerwand herangezogen.

\subsection{Zusammenfassende Darstellung der alternativen Wandsysteme}

In Tab. 4 werden die alternativen Wandsysteme zusammengefasst und die jeweiligen Wandstärken wiedergegeben. Die Alternative der Außenwand bestehend aus Stahlbetonfertigteilelement und Wärmedämmung anhand einer VIP sowie innenseitiger Verspachtelung ergibt eine Gesamtstärke von $22 \mathrm{~cm}$. Im Vergleich zum Referenzaufbau ergibt sich daraus eine Reduzierung von $20 \mathrm{~cm}$. Bei den Innenwänden lassen sich Reduzierungen bei den tragendenden Wänden von $5,0 \mathrm{~cm}$ und bei den nicht tragenden Wänden von $2,5 \mathrm{~cm}$ erzielen. Insbesondere die innovativen Bauelemente lassen eine deutliche Reduktion der Wanddicke zu. Das STB-Fertigteilelement schafft eine Einsparung von $5 \mathrm{~cm}$, während die VIP $14 \mathrm{~cm}$ einspart.

Tab. 4 Wandaufbau und Aufbaustärken [in $\mathrm{cm}$ ] alternativen Wandsysteme. (Eigene Darstellung)

\begin{tabular}{|c|c|c|c|c|c|c|}
\hline Bauteil & AW & & IW_tr & & IW_ntr & \\
\hline- & Spachtelung & $0,5 \mathrm{~cm}$ & Spachtelung & $0,5 \mathrm{~cm}$ & $\begin{array}{l}\text { Gipskartonplatten } \\
\text { (1-fach) }\end{array}$ & $1,25 \mathrm{~cm}$ \\
\hline- & $\begin{array}{l}\text { STB-Fertig- } \\
\text { teilelement }\end{array}$ & $15 \mathrm{~cm}$ & $\begin{array}{l}\text { STB-Fertig- } \\
\text { teilelement }\end{array}$ & $15 \mathrm{~cm}$ & $\begin{array}{l}\text { Installationsebene } \\
\text { (Dämmung) }\end{array}$ & $5,0 \mathrm{~cm}$ \\
\hline- & $\begin{array}{l}\text { VIP mit } \\
\text { Außenputz }\end{array}$ & $6,5 \mathrm{~cm}$ & $\begin{array}{l}\text { Vorsatzschale } \\
\text { Gipskartonplat- } \\
\text { ten }\end{array}$ & $7,5 \mathrm{~cm}$ & $\begin{array}{l}\text { Gipskartonplatten } \\
\text { (1-fach) }\end{array}$ & $1,25 \mathrm{~cm}$ \\
\hline Summe: & $22 \mathrm{~cm}$ & & $24,0 \mathrm{~cm}$ & & $7,5 \mathrm{~cm}$ & \\
\hline$\Delta r e f$ & $-20 \mathrm{~cm}$ & & $-5,0 \mathrm{~cm}$ & & $-2,5 \mathrm{~cm}$ & \\
\hline
\end{tabular}

$\Delta$ ref: Differenz zur Wandstärke des Referenzaufbaus 


\section{Wirtschaftlichkeitsberechnung und Ergebnisse}

Während für die konventionelle Bauweise auf bestehende Baukostensammlungen zurückgegriffen werden kann, bestehen für die innovativen Bauelemente keine allgemein zugänglichen Baukostensammlungen. Deshalb wurden in dieser Studie die Listenpreise von Herstellerfirmen erhoben und fehlende Aufwendungen, wie bspw. für die Montage durch Erfahrungswerte geschätzt. Zur Berechnung der Wirtschaftlichkeit einer Maßnahme wird stets vom Referenzgebäude ausgegangen. Dabei werden von der jeweiligen Maßnahme die Differenzen der Kosten und der Flächen ermittelt. Die Kostenermittlung basiert dabei auf einem Vollkostenansatz, welcher speziell bei den Außenwänden zu berücksichtigen ist. Werden Außenwandkonstruktionen in der Aufbaustärke verringert, so zieht dies auch Folgekosten im Inneren nach sich. Dies bedeutet, dass davon auch die Deckenaufbauten und Innenwände betroffen sind. Der Quotient der daraus ermittelten gesamten Kostendifferenz durch den Flächengewinn ergibt sohin den Schwellenwert pro zusätzlichen $\mathrm{m}^{2}$ NUF. Die wirtschaftliche Vorteilhaftigkeit der Maßnahme ist dann gegeben, wenn dieser Schwellenwert unter dem Angebotspreis (Verkaufspreis) pro $\mathrm{m}^{2}$ NUF liegt. Ist der Angebotspreis geringer als der Schwellenwert, wäre die Maßnahme nicht wirtschaftlich. Zusammengefasst werden die berücksichtigten Kosten und die ermittelten Schwellenwerte für die Außen- und Innenwände in den nachstehenden Gln. 1 und 2 dargestellt.

\section{Break-even/Schwellenwert für die Außenwandkonstruktionen}

$$
S W_{A W}=\frac{\Delta K_{A W}+K_{I W_{-} \mathrm{t} r}+K_{I W_{-} \mathrm{ntr}}+K_{D E}}{\mathrm{NUF}_{\mathrm{NEU}}}
$$

Legende:

$\mathrm{SW}_{\mathrm{AW}}$ : $\quad$ Schwellenwert für Außenwandkonstruktionen pro zusätzlicher Nutzungsfläche (in $€ / \mathrm{m}^{2} \mathrm{NUF}$ )

$\Delta \mathrm{K}_{\mathrm{Aw}}$ : Differenz der Kosten für die Außenwandkonstruktion zum Referenzgebäude

$\mathrm{K}_{\mathrm{IW}_{-} \mathrm{tr}}$ : Mehrkosten der tragenden Innenwandkonstruktionen

$\mathrm{K}_{\mathrm{IW} \_n t r}$ : Mehrkosten der nicht tragenden Innenwandkonstruktionen

$\mathrm{K}_{\mathrm{DE}}$ : $\quad$ Mehrkosten der Deckenaufbauten

NUF $_{\mathrm{NEU}}$ : Zusätzliche Nutzungsfläche (im Vergleich zum Referenzgebäude)

\section{Break-even/Schwellenwert für die Innenwandkonstruktionen}

$$
S W_{I W}=\frac{\Delta K_{I W_{-} \mathrm{t} r}+\Delta K_{I W_{-} \mathrm{ntr}}+K_{D E}}{\mathrm{NUF}_{\mathrm{NEU}}}
$$


Legende:

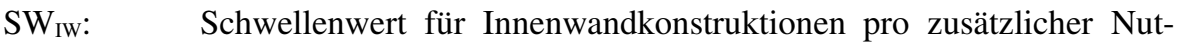
zungsfläche (in $€ / \mathrm{m}^{2} \mathrm{NUF}$ )

$\Delta \mathrm{K}_{\mathrm{IW} \_\mathrm{r}}$ : Differenz Kosten der tragenden Innenwandkonstruktionen zum Referenzgebäude

$\Delta \mathrm{K}_{\mathrm{IW} \_ \text {ntr }}$ : Differenz Kosten der nicht tragenden Innenwandkonstruktionen zum Referenzgebäude

$\mathrm{K}_{\mathrm{DE}}$ : $\quad$ Mehrkosten der Deckenaufbauten

NUF $_{\mathrm{NEU}}$ : Zusätzliche Nutzungsfläche (im Vergleich zum Referenzgebäude)

Der Unterschied in den zwei Gleichungen besteht in der Berücksichtigung der Mehrkosten von Innenwänden. Während bei der Außenwandkonstruktion die Mehrkosten für die Innenwände und auch Deckenaufbau zu berücksichtigen sind, sind bei den Innenwänden lediglich die Mehrkosten für den Deckenaufbau enthalten. Für den Deckenaufbau wurden Kosten von $87 € / \mathrm{m}^{2}$ (ohne Stahlbetondecke) bis $125 € / \mathrm{m}^{2}$ (mit Stahlbetondecke) angesetzt. Der Unterschied in der Verwendung liegt darin, dass bei Verringerung der Außenwanddicke auch die Stahlbetondecke vergrößert wird, wohin gegen bei den Innenwänden lediglich der Decken- und Fußbodenbodenaufbau zu berücksichtigen sind.

\subsection{Ergebnisse - Break-Even Point (Schwellenwert)}

In der Tab. 5 werden die jeweiligen Schwellenwerte der einzelnen Maßnahmen (AW - Außenwand, IW_tr - Innenwand tragend, IW_ntr - Innenwand nicht tragend) zusammen gefasst. Bei der Außenwand ergibt sich eine Kostendifferenz in Summe von $128.252 €$ und eine Flächendifferenz von $50,33 \mathrm{~m}^{2}$, daraus ergibt sich

Tab. 5 Ergebnisse - Kostendifferenz für die Wandkonstruktionen. (Eigene Darstellung)

\begin{tabular}{|c|c|c|c|c|}
\hline Bauteil & AW & IW_tr & IW_ntr & Gesamt \\
\hline$\overline{\Delta \mathrm{K}_{\mathrm{AW}}}$ & $118.917 €$ & - & - & $118.917 €$ \\
\hline $\mathrm{K}_{\mathrm{IW} \_\mathrm{tr}}$ & $1834 €$ & - & - & $1834 €$ \\
\hline $\mathrm{K}_{\mathrm{IW} \_n t r}$ & $1210 €$ & - & - & $1210 €$ \\
\hline $\mathrm{K}_{\mathrm{DE}}$ & $6291 €$ & $678 €$ & $665 €$ & $7634 €$ \\
\hline$\Delta \mathrm{K}_{\mathrm{IW} \_\mathrm{tr}}$ & - & $2240 €$ & - & $.240 €$ \\
\hline$\Delta \mathrm{K}_{\mathrm{IW} \_n t r}$ & - & - & $-3237 €$ & $-3237 €$ \\
\hline$N U F_{N E U}$ & $50,33 m^{2}$ & $7,79 m^{2}$ & $7,64 m^{2}$ & $65,76 m^{2}$ \\
\hline$S W_{A W / I W}$ & $2548 € / m^{2} N U F$ & $374 € / m^{2} N U F$ & $-337 € / m^{2} N U F$ & $1956 € / \mathrm{m}^{2} \mathrm{NUF}$ \\
\hline
\end{tabular}

Tab. 6 Ergebnisse - Grundflächen und Kennwerte. (Eigene Darstellung)

\begin{tabular}{lll}
\hline Grundflächen und Kennwerte & Referenz & Alternative \\
\hline Brutto-Grundfläche (BGF) & $1173,21 \mathrm{~m}^{2}$ & $1173,21 \mathrm{~m}^{2}$ \\
Nutzungsfläche (NUF) & $849,62 \mathrm{~m}^{2}$ & $914,83 \mathrm{~m}^{2}$ \\
Konstruktions-Grundfläche (KGF) & $206,85 \mathrm{~m}^{2}$ & $141,64 \mathrm{~m}^{2}$ \\
NUF/BGF & 0,724 & 0,780 \\
KGF/BGF & 0,176 & 0,121 \\
\hline
\end{tabular}


ein Schwellenwert von $2548 € / \mathrm{m}^{2}$ zusätzlicher NUF. Bei den Innenwänden sind die Schwellenwerte geringer und bei den nicht tragenden Innenwänden gehen diese Aufwendungen sogar in den negativen Bereich. Dies kommt daher, dass die alternative Konstruktion zur Referenzkonstruktion niedrigere Kosten aufweist.

Fasst man alle drei Maßnahmen zusammen, so ergeben sich Mehrkosten in der Höhe von $\sim 128.600 €$. Dem gegenüber ergibt sich ein Flächengewinn von $65,76 \mathrm{~m}^{2}$ Nutzungsfläche. Dies entspricht einer Steigerung der Nutzungsfläche gegenüber dem Referenzobjekt von 7,7\%. Setzt man die Mehrkosten von $\sim 128.600 €$ ins Verhältnis zum Flächengewinn von $65,76 \mathrm{~m}^{2}$, so ergibt dies $1956 € / \mathrm{m}^{2}$ zusätzlicher NUF. Es ist ersichtlich, dass die Maßnahme der Außenwände den größten Effekt bei der Flächendifferenz erzielt, jedoch haben die Innenwände aufgrund der geringeren Aufwendungen eine höhere Kosteneffizienz. Die Tab. 6 stellt die Grundflächen und Kennwerte der Referenz denen der Alternative gegenüber.

Betrachtet man das ermittelte Verhältnis der KGF/BGF der Alternative mit 12,1\%, so liegt dieser Wert unter den eingangs erwähnten Kennwerten, die im Minimum mit 14,6\% (BKI Baukosteninformationszentrum 2017) beziffert sind.

\subsection{Einflussanalyse - Ergebnisse aus der Simulation}

Aufgrund der geringen Datenlage der Kostenkennwerte und der Erhebung der Einflussgrößen der Geometrie wie auch der Kostenkennwerte wurde eine Simulation durchgeführt. Die Simulation erfolgte mittels @ risk (Palisade Corporation). Die Verteilungen der Eingangsparameter wurden risikoavers festgelegt. Für die Kostenkennwerte wurden als Minimum die Ausgangsdaten, der Mittelwert mit $+10 \%$ und das Maximum mit $+20 \%$ der Ausgangsdaten angenommen, in Form einer Dreiecksverteilung. Die Bandbreite der geometrischen Kennwerte (AwFl/BGF, IwFl/BGF) erfolgte auf Grundlage des Referenzgebäudes und der Kennwerte aus dem BKI. Die Kennwerte des Referenzgebäudes wurden dabei durchgehend als Maximum Wert definiert, die Minimum Werte dem BKI entnommen. Als Verteilung wurde hierbei

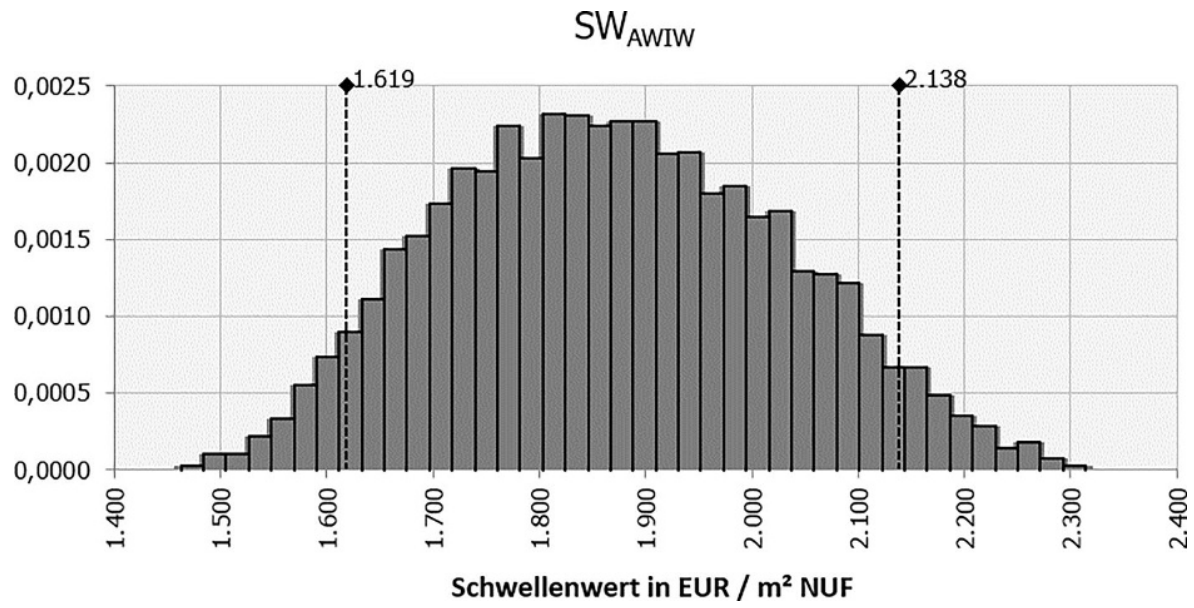

Abb. 3 Ergebnisse der Simulation. (Eigene Darstellung) 
Tab. 7 Eingangsparameter der Simulation. (Eigene Darstellung)

\begin{tabular}{|c|c|c|c|c|}
\hline Parameter & Diagramm & Min & Mittelwert & $\operatorname{Max}$ \\
\hline \multirow[t]{2}{*}{ AwFl_ko } & & 423 & 488 & 552 \\
\hline & 423 & & & \\
\hline \multirow[t]{2}{*}{ IwFl_tr } & & 270 & 312 & 355 \\
\hline & 270 & & & \\
\hline \multirow[t]{2}{*}{ IwFl_ntr } & & 633 & 731 & 830 \\
\hline & 633 & & & \\
\hline$\Delta \mathrm{K}_{\mathrm{AW}} / \mathrm{m}^{2}$ AwFl_ko & & 200 & 230 & 260 \\
\hline$\Delta \mathrm{K}_{\mathrm{IW} \_\mathrm{tr} / \mathrm{m}^{2} \mathrm{IwFl} \text { _tr }}$ & & 6 & 7,5 & 9 \\
\hline$\Delta \mathrm{K}_{\mathrm{IW} \_n t r} / \mathrm{m}^{2} \mathrm{IwFl} \_\mathrm{ntr}$ & & -4 & -3 & -2 \\
\hline
\end{tabular}

eine Uniform Verteilung angenommen. Sämtliche Eingangsparameter inkl. deren Bandbreite und Verteilung können Tab. 7 entnommen werden.

Die Simulation erfolgte anhand von 10.000 Iterationen. Die sich daraus ermittelten Ergebnisse in Form einer Häufigkeitsverteilung sind in Abb. 3 dargestellt. Aus der Simulation ergibt sich für den Schwellenwert (SW) pro zusätzlichem $\mathrm{m}^{2}$ NUF ein Mittelwert von $1870 € / \mathrm{m}^{2}$. Die Bezeichnung $\mathrm{SW}_{\mathrm{Aw}}$ iw in Abbildung beinhaltet die Parameter der Außen- und Innenwand. Die in der Abbildung angegebenen Werte $1619 € / \mathrm{m}^{2}$ NUF und $2138 € / \mathrm{m}^{2}$ NUF beziehen sich auf die $5 \%$ bzw. $95 \%$ Perzentile. Das Minimum liegt bei $1463 € / \mathrm{m}^{2}$ NUF und das Maximum bei $2314 € / \mathrm{m}^{2} \mathrm{NUF}$.

Aus der Einflussanalyse geht hervor, dass die Parameter Fläche und Kosten der Außenwand (AW) den größten Einfluss auf den Schwellenwert als Ergebnis haben. Die Parameter der Innenwände haben einen weit geringeren Effekt bzw. Potential. Die Einflüsse der jeweiligen Parameter können dem Tornadodiagramm der Abb. 4 entnommen werden. 


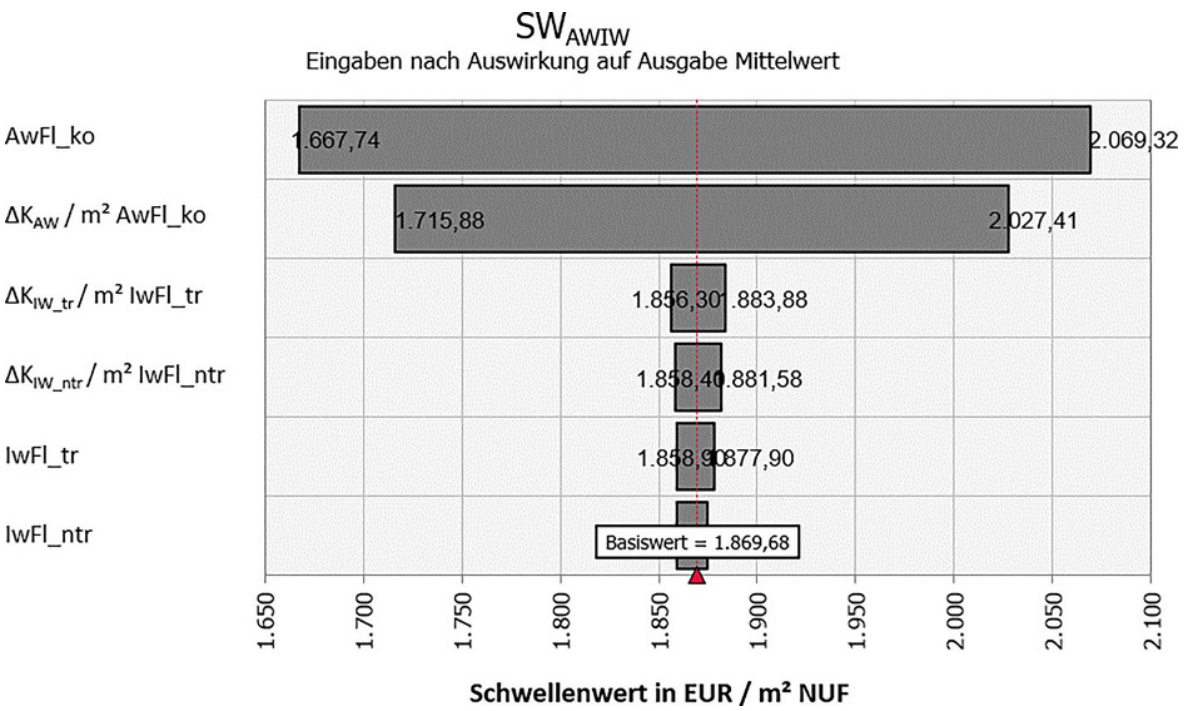

Abb. 4 Einflussanalyse der Parameter. (Eigene Darstellung)

\section{Zusammenfassung und Diskussion}

Aus der Untersuchung geht zusammengefasst hervor, dass alternative und innovative Materialien in den Wandaufbauten ein wirtschaftliches Potential darstellen. Die Gegenüberstellung am Referenzgebäude zeigt, dass unter der Annahme der vollen Ausreizung der BGF eine Erhöhung der Nutzungsfläche um 7,7\%, absolut ca. $65 \mathrm{~m}^{2}$, durch die alternativen Wandaufbauten möglich ist. Bei den analysierten Maßnahmen bieten die Außenwände ein wesentlich höheres Potential in der Generierung zusätzlicher Fläche. Die gewonnene Fläche aus den Innenwänden ist jedoch wirtschaftlicher als jene der Außenwände, da in diesem Fall keine zusätzlichen Kosten entstehen, d.h. es sind keine teuren Materialien wie die VIP erforderlich. Bei den Außenwänden mit VIP gilt zu beachten, dass in dieser Untersuchung eine Variante mit Außenputz berücksichtigt wurde. Weitere Varianten, wie bspw. in Form von hinterlüfteten Konstruktionen wurden aufgrund des Minimal-Prinzips der Kosten und der Aufbaustärke nicht betrachtet. Die ermittelten Ergebnisse als Schwellenwert pro $\mathrm{m}^{2}$ zusätzlicher Nutzungsfläche von $1956 €$ zeigen, dass diese im Vergleich zu den aktuell am Markt bestehenden Verkaufspreisen wirtschaftlich vielerorts von Vorteil sind. Als Diskussion sollten die Aspekte der Qualität angesprochen werden. Gerade im Hinblick auf die gewählte Konstruktion der nicht tragenden Innenwände, die zwar den gesetzlichen Anforderungen genügen, ist deren bauphysikalische Einordnung hinsichtlich der heutigen kundenseitigen Anforderungen (Akustik und Raumklima) zu hinterfragen. Des Weiteren ist zu erwähnen, dass eventuelle zusätzliche Aufwendungen im Bereich der Haustechnik und Leitungsführung hier nicht berücksichtigt wurden. Abschließend kann gesagt werden, dass derartige innovative Materialien für den Hochbau eine gute Alternative darstellen können. Aktuell fehlt es in der praktischen Anwendung noch an Erfahrungswerten zur Etablierung solcher Varian- 
ten. Speziell im Neubau könnten sich durch die Vorfertigung, evtl. in Verbindung mit der seriellen Bauweise, interessante Verwendungsmöglichkeiten ergeben. In diesem Zusammenhang könnten technische, physikalische oder wirtschaftliche Untersuchungen als Anknüpfungspunkte weiterführender Forschung sein. Darüber hinaus wären auch Untersuchungen aus dem Gebiet der Wahrnehmung und Kognition von Relevanz, um Erkenntnisse der kundenseitigen Wahrnehmung von Anforderungen bis hin zur Zahlungsbereitschaft für spezielle Konstruktionen (bspw. ökologische) zu identifizieren.

Open Access Dieser Artikel wird unter der Creative Commons Namensnennung 4.0 International Lizenz (http://creativecommons.org/licenses/by/4.0/deed.de) veröffentlicht, welche die Nutzung, Vervielfältigung, Bearbeitung, Verbreitung und Wiedergabe in jeglichem Medium und Format erlaubt, sofern Sie den/die ursprünglichen Autor(en) und die Quelle ordnungsgemäß nennen, einen Link zur Creative Commons Lizenz beifügen und angeben, ob Änderungen vorgenommen wurden.

\section{Literatur}

BKI Baukosteninformationszentrum (2010) BKI Baukosten 2010 Teil 1: Statistische Kostenkennwerte für Gebäude. Baukosteninformationszentrum Deutscher Architektenkammern GmbH, Stuttgart

BKI Baukosteninformationszentrum (2017) Baukosten Gebäude Neubau: Statistische Kostenkennwerte. Baukosteninformationszentrum Deutscher Architektenkammern GmbH, Stuttgart

Deutsches Institut für Normung (2016) Grundflächen und Rauminhalte im Bauwesen - Teil 1: Hochbau. DIN 277. Beuth, Berlin

Gubbels F, Santi DD, Baily V (2015) Durability of vacuum insulation panels in the cavity of an insulating glass unit. J Build Phys 38:485-499. https://doi.org/10.1177/1744259114522118

Jelle BP (2011) Traditional, state-of-the-art and future thermal building insulation materials and solutions-properties, requirements and possibilities. Energy Build 43:2549-2563. https://doi.org/10. 1016/j.enbuild.2011.05.015

Johansson P (2012) Vacuum insulation panels in buildings. Literature review. Report, Department of Civil and Environmental Engineering, Chalmers University of Technology, Schweden, S 1-27. http:// publications.lib.chalmers.se/records/fulltext/155961.pdf. Zugegriffen: 13. Apr. 2018

Kalusche W (2011) Grundflächen und Planungskennwerte von Wohngebäuden. In: Gralla M, Sundermeier M (Hrsg) Innovation im Baubetrieb. Werner, Köln, S 35-48 (https://www-docs.tu-cottbus.de/ bauoekonomie/public/Forschung/Publikationen/Kalusche-Wolfdietrich/2011/Udo_Blecken_Grundfl \%C3\%A4chen_Wohnbauten.pdf. Gesehen 13.04.2018)

Kolbitsch A, Stalf-Lenhardt ML, Kropik A, Prestros L (2008) Studie über Wirtschaftlichkeitsparameter und einen ökonomischen Planungsfaktor für geförderte Wohnbauprojekte in Wien, Wien. https://www.wko.at/branchen/gewerbe-handwerk/bau/Langfassung_Studie_.pdf. Zugegriffen: 13. Apr. 2018

Krass J, Mitransky B, Rupp G (2009) Baustoffe. In: Krass J, Mitransky B, Rupp G (Hrsg) Grundlagen der Bautechnik. Vieweg+Teubner, Wiesbaden, S 213-430

McKeen P, Fung AS (2014) The effect of building aspect ratio on energy efficiency: a case study for multiunit residential buildings in Canada. Buildings 4:336-354. https://doi.org/10.3390/buildings4030336

Naji S, Çelik OC, Johnson Alengaram U et al (2014) Structure, energy and cost efficiency evaluation of three different lightweight construction systems used in low-rise residential buildings. Energy Build 84:727-739. https://doi.org/10.1016/j.enbuild.2014.08.009

Palisade Corporation (2017) @RISK Risk Analysis Software mit Hilfe von Monte-Carlo-Simulation für Excel

Pfau J (2013) Wände in Trockenbauweise. In: Nabil FA (Hrsg) Lehrbuch der Hochbaukonstruktionen, 4. Aufl. Springer, Wiesbaden, S 837-891

Rupp G (2009) Nichttragende innere Trennwände. In: Rupp G (Hrsg) Technologie für Stuckateure und Trockenbauer, 4. Aufl. Vieweg+Teubner, Wiesbaden, S 231-290

Schwab H, Stark C, Wachtel J et al (2005) Thermal bridges in vacuum-insulated building facades. J Therm Envel Build Sci 28:345-355. https://doi.org/10.1177/1097196305051794 
Sev A, Zgen A (2009) Space efficiency in high-rise office buildings. METU J Fac Archit 26(2):69-89. https://doi.org/10.4305/METU.JFA.2009.2.4

Tenpierik MJ, Cauberg JJM (2010) Encapsulated vacuum insulation panels: theoretical thermal optimization. Build Res Inf 38:660-669. https://doi.org/10.1080/09613218.2010.487347

Wakili KG, Bundi R, Binder B (2004) Effective thermal conductivity of vacuum insulation panels. Build Res Inf 32:293-299. https://doi.org/10.1080/0961321042000189644

Yamankaradeniz N (2015) Minimization of thermal insulation thickness taking into account condensation on external walls. Adv Mech Eng 7:1-11. https://doi.org/10.1177/1687814015604803 